ACCEPTED FOR APJ

Preprint typeset using $\mathrm{LAT}_{\mathrm{E}} \mathrm{X}$ style emulateapj v. 11/12/01

\title{
CLUSTER HEATING BY VISCOUS DISSIPATION OF SOUND WAVES
}

\author{
Mateusz Ruszkowski ${ }^{1}$ \\ JILA, Campus Box 440, University of Colorado at Boulder, CO 80309-0440; \\ mr@quixote.colorado.edu \\ MARCUS BRÜGGEN \\ International University Bremen, Campus Ring 1, 28759 Bremen, Germany; \\ m.brueggen@iu-bremen.de \\ AND \\ Mitchell C. Begelman ${ }^{2}$ \\ JILA, Campus Box 440, University of Colorado at Boulder, CO 80309-0440; \\ mitch@jila.colorado.edu \\ accepted for ApJ
}

\begin{abstract}
We simulate the effects of viscous dissipation of waves that are generated by AGN activity in clusters of galaxies. We demonstrate that the amount of viscous heating associated with the dissipation of these waves can offset radiative cooling rates in cooling flow clusters of galaxies. This heating mechanism leads to spatially distributed and approximately symmetrical dissipation. The heating waves reach a given distance from the cluster center on a timescale shorter than the cooling time. This means that this heating mechanism has the potential of quenching cooling flows in a quasi-stable fashion. Moreover, the heating is gentle as no strong shocks are present in the simulations. We first investigated whether a single continuous episode of AGN activity can lead to adequate dissipation to balance cooling rates. These simulations demonstrated that, whereas secondary waves generated by the interaction of the rising bubble with the intracluster medium are clearly present, viscous heating associated with the dissipation of these waves is insufficient to balance radiative cooling. It is only when the central source is intermittent that the viscous dissipation of waves associated with subsequent episodes of activity can offset cooling. This suggests that the ripples observed in the Perseus cluster can be interpreted as being due to the AGN duty cycle, i.e., they trace AGN activity history. The simulations were performed using the PPM adaptive mesh refinement code FLASH in two dimensions.
\end{abstract}

Subject headings: cooling flows-galaxies: active - waves

\section{INTRODUCTION}

Cooling timescales of gas in the central regions of clusters of galaxies are often much shorter than the Hubble time. Initially, this led to suggestions that the intracluster medium (ICM) is flowing into the cluster center at rates of up to $1000 M_{\odot} /$ yr. However, recent XMM Newton and Chandra observations suggest that the actual inflow rates are much smaller than expected, and that feedback from active galactic nuclei (AGN) may play a crucial role in regulating mass accretion rates (e.g., Fabian et al. (2000, 2002); McNamara et al. (2000); Blanton et al. (2001); Churazov et al. (2002)). The significance of AGN feedback is supported by the observation that about $70 \%$ of $\mathrm{cD}$ galaxies in cluster centers show evidence for active radio sources (Burns 1990). The advantage of the AGN heating model over other models is that the heating is supplied near the cluster center where the cooling flow problem is most severe. For example, AGN heating may explain why the gas temperature, while declining towards cluster centers, does not drop below about $1 \mathrm{keV}$ (Peterson et al. 2001, 2003; Tamura et al. 2001).

AGN are believed to be intermittent with an intermittency period of $10^{5}-10^{8} \mathrm{yr}$, much shorter than the Hubble time and shorter than or comparable to the central cooling time (e.g., Mazzotta et al. (2002); Croom et al. (2003); Fabian et al. (2003a,b)). Therefore, one expects that AGN-heated cooling flows could be stabilized in a timeaveraged sense and that "cooling catastrophes" could be prevented. Recent observations of ripples and weak shocks in the Perseus cluster (Fabian et al. 2003a,b) and the Virgo cluster (Forman et al. 2003) provide observational support for this idea. Fabian et al. (2003a) were the first to show that viscous dissipation of these waves is sufficient to offset radiative cooling in the Perseus cluster.

Recently, several studies have addressed the problem of AGN heating of clusters from a numerical perspective. These studies can be divided into two main categories depending on the parameter regime considered: models in which the mechanical energy supply to the cluster is momentum driven (e.g., Tabor \& Binney (1993); Reynolds et al. (2001)) and those in which it is buoyancy driven (e.g., Churazov et al. (2001); Brüggen et al. (2002); Brüggen \& Kaiser (2002); Brüggen (2003); Quilis et al. (2001)). In this paper we focus on the latter regime. An alternative idea was proposed by Pringle (1989), who suggested that clusters can be heated by dissipation of sound waves generated by galaxy motions in the cluster. Further support

1 Chandra Fellow

2 also at Department of Astrophysical and Planetary Sciences, University of Colorado at Boulder 
for the idea that viscosity may play an important role in the intracluster medium comes from the recent study of density profiles in clusters (Hansen \& Stadel 2003). The main purpose of this paper is to demonstrate that clusters can be heated efficiently by wave dissipation associated with activity of AGN located in their centers. Although our simulations are two-dimensional and therefore not directly applicable to real clusters, we argue that the basic result should be preserved in three dimensions.

\section{ASSUMPTIONS OF THE MODEL}

\subsection{Initial Conditions}

The intracluster medium is initially assumed to be in hydrostatic equilibrium in an NFW potential (Navarro et al. 1995,1997$)$ for which the gravitational acceleration as a function of the distance from the cluster center $r$ is given by

$$
\mathbf{g}(r)=4 \pi G \rho_{\mathrm{crit}} \delta_{c} r_{c} x^{-2}\left[-\ln (1+x)+\frac{x}{1+x}\right] \hat{\mathbf{r}},
$$

where $r_{c}=100 \mathrm{kpc}$ is the core radius, $x=r / r_{c}, \hat{\mathbf{r}}=\mathbf{r} / r$, $\delta_{c}=3.0 \times 10^{4}$ is the central overdensity, and $\rho_{\text {crit }}=$ $3 H_{o}^{2} /(8 \pi G)$ is the critical density of the Universe (we assume $\left.H_{o}=75 \mathrm{~km} \mathrm{~s}^{-1} \mathrm{Mpc}^{-1}\right)$. The initial temperature distribution is given by

$$
T(r)=T_{o}\left(1+\frac{r}{r_{o}}\right)^{\beta}
$$

where $T_{o}=3.0 \mathrm{keV}, r_{o}=10 \mathrm{kpc}$ and $\beta=0.22$. The temperature at $100 \mathrm{kpc}$ is $5.1 \mathrm{keV}$. The central electron number density is $2.8 \times 10^{-2} \mathrm{~cm}^{-3}$. The electron number density at $100 \mathrm{kpc}$ is approximately $5.3 \times 10^{-3} \mathrm{~cm}^{-3}$. This corresponds to a central cooling time of $\sim 1.3 \times 10^{9}$ years and a cooling time of $\sim 1.0 \times 10^{10}$ years at 100 $\mathrm{kpc}$. These are values typical of a cooling flow cluster as the central cooling time is much shorter than the Hubble time. Note that we do not intend to model the details of any particular cluster even if our studies have been motivated by observations of Perseus. Since our simulations are two-dimensional, a quantitative comparison is not possible. For one thing, the wave energy density can be expected to decay more slowly with radius in two dimensions $\left(\propto r^{-1}\right)$ than in three $\left(\propto r^{-2}\right)$. Moreover, bubbles expanding into two dimensions are expected to be larger than equivalent bubbles expanding three-dimensionally; indeed, for our initial conditions chosen to approximate those in Perseus, we find that our computed bubbles are larger than the observed cavities.

We assume that the gas is fully ionized and characterized by $X=0.75$ and $Y=0.25$, where $X$ and $Y$ are the hydrogen and helium fractions. The injected gas is characterized by an adiabatic index $\gamma_{\text {bubble }}=4 / 3$, whereas for the ambient gas we used $\gamma_{\mathrm{ICM}}=5 / 3$. Calculations were done in two dimensions for 9 levels of refinement using the PPM adaptive mesh refinement code FLASH. The size of the computational domain was $(200 \mathrm{kpc})^{2}$. Thus, the effective resolution in our simulations was $2048^{2}$ zones, which corresponds to $\sim 0.1 \mathrm{kpc}$. We have performed convergence tests and found that neither the geometry of the bubbles nor that of the waves depends on the adopted resolution. This is due to the fact that, for the parameters considered, the Reynolds number corresponding to bubbles and waves is low and also because numerical dissipation of PPM codes is known to be relatively low. The effective Reynolds numbers achievable in the simulation are proportional to the number of grid points across the fluctuation of interest to the power $n$, where $n=3$ is the order of the numerical scheme ${ }^{3}$ (Sytine et al. 2000; Balbus et al. 1996; Porter \& Woodward 1994). We used a redefined system of units in which all variables apart from temperature are close to unity and adopted outflow boundary conditions. We have also repeated our simulations in two-dimensional cylindrical coordinates and found that, in spite of unphysical effects near the symmetry axis, our conclusions remain unaffected.

\subsection{Heating}

We model AGN heating by injecting hot gas into two regions of radius $1 \mathrm{kpc}$ located $10 \mathrm{kpc}$ to either side of the cluster center. The energy injection rate, $L$, for each source and the mass injection rate per unit volume, $\dot{\rho}$, are both constant. Thus, the energy injection rate per unit mass $\dot{\epsilon}$ is computed from

$$
\dot{\epsilon}=\left(\frac{L}{\rho V}-\epsilon \frac{\dot{\rho}}{\rho}\right)
$$

where $V$ is the volume of one injection region (of radius $1 \mathrm{kpc}$ ). We used $L=1.5 \times 10^{43} \mathrm{erg} \mathrm{s}^{-1}$ and $\dot{\rho} V=0.01$ $\mathrm{M}_{\odot} \mathrm{yr}^{-1}$. The energy injection is intermittent with an intermittency period of $3 \times 10^{7}$ years, i.e., the source is active for $1.5 \times 10^{7}$ years and dormant for $1.5 \times 10^{7}$ years. In the initial state for each activity episode, the temperature and density are a hundred times higher and lower, respectively, than the temperature and density in the initial unperturbed state at the same location.

The dissipation of mechanical energy due to viscosity, per unit mass of the fluid, was calculated from (Batchelor 1967; Shu 1992)

$$
\dot{\epsilon}_{\mathrm{visc}}=\frac{2 \mu}{\rho}\left(e_{i j} e_{i j}-\frac{1}{3} \Delta^{2}\right),
$$

where $\Delta=e_{i i}$ and

$$
e_{i j}=\frac{1}{2}\left(\frac{\partial v_{i}}{\partial x_{j}}+\frac{\partial v_{j}}{\partial x_{i}}\right)
$$

and where $\mu$ is the dynamical coefficient of viscosity. We use the standard Spitzer viscosity (Braginskii 1958), for which $\mu=1.1 \times 10^{-16} T^{5 / 2} \mathrm{~g} \mathrm{~cm}^{-1} \mathrm{~s}^{-1}$. As conditions inside the buoyantly rising bubbles are very uncertain and because we want to focus on energy dissipation in the ambient ICM, we assume that dissipation occurs only in the regions surrounding the buoyant gas. To this end we impose a condition that switches on viscous effects provided that the fraction of the injected gas in a given cell is much smaller than unity. We point out that the value of viscosity in the ICM, just as any other transport parameters

${ }^{3}$ See, e.g., Bowers \& Wilson (1991) for the definition of "the order of the numerical scheme", as it is different from the customary definition of accuracy of a perturbative calculation. 
such as, e.g., thermal conduction, is highly uncertain, and especially the role of magnetic fields is unclear.

We implemented a fully compressible version of the viscous velocity diffusion equation in the FLASH code. Velocity diffusion was simulated by solving the momentum equation

$$
\frac{\partial\left(\rho v_{i}\right)}{\partial t}+\frac{\partial}{\partial x_{k}}\left(\rho v_{k} v_{i}\right)+\frac{\partial P}{\partial x_{i}}=\rho g_{i}+\frac{\partial \pi_{i k}}{\partial x_{k}}
$$

where

$$
\pi_{i k}=\frac{\partial}{\partial x_{k}}\left[2 \mu\left(e_{i k}-\frac{1}{3} \Delta \delta_{i k}\right)\right]
$$

and where all other symbols have their usual meaning.

\subsection{Cooling}

We switched off radiative cooling because the initial cooling time in the center is longer than the overall duration of the simulation. However, we calculate the radiative cooling rates in order to compare them with the viscous heating rates. For this purpose we use the fit to the cooling function by Tozzi \& Norman (2001), which is based on detailed calculations by Sutherland \& Dopita (1993)

$$
n_{e}^{2} \Lambda=\left[C_{1}\left(k_{B} T\right)^{\alpha}+C_{2}\left(k_{B} T\right)^{\beta}+C_{3}\right] n_{i} n_{e},
$$

where $n_{i}$ is the ion number density and the units for $k_{B} T$ are $\mathrm{keV}$. For an average metallicity $Z=0.3 Z_{\odot}$, the constants in equation (8) are $\alpha=-1.7, \beta=0.5, C_{1}=$ $8.6 \times 10^{-3}, C_{2}=5.8 \times 10^{-2}$ and $C_{3}=6.4 \times 10^{-2}$ and we can approximate $n_{i} n_{e}=(X+0.5 Y)(X+0.25 Y)\left(\rho / m_{p}\right)^{2}$. The units of $\Lambda$ are $10^{-22}$ erg $\mathrm{cm}^{3} s^{-1}$.

\section{RESULTS AND CONCLUSIONS}

The top panels in Figure 1 show a time sequence of density maps. One can observe that the gas rises subsonically in the cluster atmosphere and spreads out laterally. No strong shocks are present in this simulation, which implies that heating is gentle in agreement with Chandra observations. Density waves have maximum amplitudes of up to about 20 to 30 per cent close to the cluster center $(\sim 20$ $\mathrm{kpc}$ ) and decrease as the waves propagate outward.

The bottom panels in Figure 1 present the evolution of the viscous dissipation rate. Heating waves generated by subsequent AGN activations are clearly visible and the energy dissipated in these waves is spatially distributed in a relatively symmetric manner. The timescale for the wave pattern to reach a particular region is shorter than the local cooling time. Thus, this heating mechanism meets at least one of the basic requirements for this model to be able to reach a quasi-steady state. We note that the wave fronts propagate at slightly above the sound speed (Mach number $\sim 1.25$; faster than the buoyantly rising bubbles) as can be seen from Figure 2 by dividing the radius of an annulus by the time it takes for the wave reach it.

It is quite likely that more than one ripple is generated per episode of AGN activity. That is, subsequent outbursts may occur when the bubble has not yet settled down from the previous outburst and it still overpressured, leading to complex time-dependence. We assumed that the radio source is intermittent on a time scale of
$1.5 \times 10^{7}$ yrs. This simplified assumption on the behavior of the source reproduces two phenomena: (i) the inflation of two well-defined cavities from the cumulative effects of multiple outbursts and (ii) the production of a number of ripples or density-waves that propagate radially outward at the speed of sound, as the pressure pulse from each outburst inflates the expanding cavity slightly (see Fig. 1). The fragmentary, scalloped appearance of the cavities and sound waves is probably overemphasized because the simulation is two-dimensional. The small-scale structure would presumably be suppressed in a three-dimensional model viewed in projection onto the plane of the sky.

Note that the waves disperse as they propagate away from the center. This dispersion is almost entirely due to explicit velocity diffusion, as tests without this effect have demonstrated. We stress that our use of the Spitzer viscosity is meant to be illustrative and may not accurately represent momentum transport in the magnetized intracluster medium. For one thing, magnetic shear stress is likely to dominate over molecular viscosity in the transport of bulk momentum. This could either enhance or suppress the dissipation of sound wave, and will almost certainly make the dependence of stress on the velocity field more complicated. For another, in this macroscopic form of momentum transport the rate of dissipation (due to reconnection) is nonlocally related to the stress tensor. Treatment of these effects will require high-resolution magnetohydrodynamical simulations. Moreover, magnetic fields could introduce effects similar to bulk viscosity, as a result of plasma microinstabilities. In our simulations we neglected bulk viscosity, since it vanishes for an ideal gas. We note that bulk viscosity, if present, could dissipate waves even more efficiently. Finally, we have neglected the effects of thermal conduction, which (assuming Spitzer conductivity) could damp the sound waves more quickly than Spitzer viscosity (since the conductive dissipation exceeds viscous one by a factor $\sim 10$ under simplified assumption that waves are plane and linear and that the gas has constant density and pressure and gravity can be neglected (Landau \& Lifshitz 1975)). Since conductivity is expected to be suppressed by magnetic fields, a realistic assessment of whether conduction enhances the damping rate of sounds waves is beyond the scope of this investigation.

Recognizing these caveats, in Figure 2 we compute the ratio of the viscous heating rate to the radiative cooling rate as a function of time, averaged over a series of concentric annuli around the cluster center. As the waves need more time to reach the gas located further away from the center, the heating rate rises at progressively later times for more distant annuli. Once the first wave has reached a given distance, viscous heating becomes comparable to the cooling rate. This is consistent with heating rate predictions made by Fabian et al. (2003a), also assuming Spitzer viscosity. We also note that dissipating waves of greater initial amplitude in our simulations would give even more heating to offset cooling. Interestingly, the average ratio of heating to cooling seems to be relatively stable as a function of time. We have also computed the volumeintegrated heating and cooling rates and found that their ratio converges to a value of the order of a few. However, the balance of heating and cooling is not automatic as it depends on the choice of parameters (e.g., AGN power and 
Ruszkowski et al.

density gradient in the intracluster medium) and here feedback may play a role. Note that the curves display a pronounced periodic behavior. This reflects the intermittency of the central source, with on- and off-states of $1.5 \times 10^{7}$ years. This is consistent with the observational estimates based on Chandra observations of ripples in the Perseus cluster (Fabian et al. 2003a,b). We performed a series of numerical experiments to investigate whether a single AGN outburst can generate waves for which the dissipation rates could offset local radiative cooling rates. These simulations demonstrated that, whereas secondary waves generated by the interaction of the rising bubble with the surrounding intracluster medium are clearly present, the viscous heating associated with a single outburst is insufficient to balance radiative cooling. This suggests that the ripples observed in the Perseus cluster can be interpreted as being due to the AGN duty cycle, i.e., they trace AGN activity history.

The work done by the expanding cavities on the ambient medium is limited to a modest fraction of the energy injected by the AGN. If the cavities are approximately in pressure balance with their surroundings, the increase in the energy of the ambient gas, $d U=d(P V) /(\gamma-1)$, is related to the work done, $d W=P d V$, by $d U \simeq d W /(\gamma-1)$. The first law of thermodynamics then implies that $d W \simeq$ $\frac{\gamma-1}{\gamma} d Q$, where $d Q$ is the heat injected into the cavity. This means that, depending on the effective value of $\gamma$ (which can range between $4 / 3$ and $5 / 3$ ), $25-40 \%$ of the energy input can be transferred to the ambient medium (see also, e.g., Churazov et al. 2001). The fraction of the input power transferred to the ICM will be larger if the cavities are overpressured. The fraction of this work that goes into acoustic energy, as opposed to other types of disturbance (e.g., g-modes or internal waves), depends on the timescale of pressure fluctuations, as well as detailed structure of the cavity-ICM interface. We expect the production of sound waves to be efficient when the AGN duty cycle is of the same order as the sound crossing time at the cavity radius, or shorter. This condition is satisfied for our chosen duty cycle of $3 \times 10^{7}$ yr. In addition to work done by in situ expansion of the cavities, a roughly comparable amount of energy is transferred to the surrounding medium, in the form of kinetic and gravitational potential energy, as the cavities rise through the backgound pressure gradient. The latter is the generic mechanism appealed to by Begelman (2001) and Ruszkowski \& Begelman (2002) in their discussion of "effervescent heating". Energy injected in this way can also be converted to heat through viscous dissipation. Our simulations map the total viscous dissipation rate, and do not distinguish between dissipation of sound waves and other kinds of motion. Note, however, that sound waves have larger propagation speeds than other modes, and therefore should progressively dominate the energetics at radii well outside the cavities.

Although we have devised a specific model in which the viscous dissipation rate of sound waves roughly balances local radiative cooling, such a balance may not be a universal property of AGN heating in cluster cores. The distribution of sound energy dissipation is largely determined by the radial structure of the model. The sound dissipation length for a fixed wavelength $L(\lambda, r)$ for the parameters in our simulations decreases from the center to the edge of the simulated region, mainly due to the decrease in density (Fabian et al. 2003). For the parameters chosen in our simulation the characteristic dissipation length near the outer edge of the grid is of order the size of the simulation region, implying that the dissipation is spread over a volume that far exceeds that of the bubbles, and much of the acoustic energy goes into heating. The steady rate of production of acoustic energy then leads to a rough balance between heating and cooling, given the adopted density and temperature profile. These conditions may not be satisfied in all clusters. As the sound dissipation length is proportional to the square of the period of the sound waves, more frequent outbursts should lead to more centrally concentrated damping. However, the dissipation rate does not depend on the AGN intermittency period as such, since the pressure pulses generated by the bubbles are likely to be far from sinusoidal and will contain a wide range of frequencies. The dispersion of the waves as they propagate suggest that the "effective" wavelength will increase with $r$, an effect that will partially counteract the decrease of $L(\lambda, r)$ with $r$.

Where the velocity field has small-scale structure or where the damping rate is much larger than the Spitzer rate, acoustic waves (as well as gravity and internal waves) can be dissipated much closer to the sites where they are generated. Distributed heating would then occur only after the bubbles had penetrated most of the cluster. This is the situation envisaged by Begelman (2001) and Ruszkowski \& Begelman (2002) in the effervescent heating scenario. This form of heating may be occurring concurrently with the large-scale acoustic heating in Perseus, and may dominate the heating in other clusters (e.g., those with smaller acoustic energy generation due to the intermittency properties of the central AGN). Note that viscosity may help the bubbles penetrate to large distances without excessive mixing.

We stress that our two-dimensional simulations do not accurately represent the behavior of three-dimensional acoustic heating in several respects. In three dimensions the energy flux in the sound waves decays faster than in the Cartesian two-dimensional case. Thus, our 2D simulation might give a more radially distributed heating rate $\left(\propto r^{-1}\right)$ compared to a 3D calculation (where the wave energy flux scales as $\propto r^{-2}$ ). However, since the dissipation rate per unit mass is inversely proportional to density $n$ while the cooling rate per unit mass is $\propto n$, a slight steepening of the density profile (by $\sim r^{-1 / 2}$ ) should compensate for the extra power of $r^{-1}$ in the energy flux. Note that the scaling of heating and cooling with density does not necessarily imply instability. The densest central regions, which cool the fastest, are in fact heated more effectively because the velocity fluctuations are stronger in the cluster core. The amount of energy injected to the cluster should also be regulated by the central cooling rate. That is, increased cooling rate should lead to more accretion onto the central AGN. Accretion of gas onto the center would then cause AGN outbursts leading to a reduced central cooling rate. If acoustic heating is truly able to stabilize radiative cooling, then the density and the luminosity of the central AGN should adjust automatically, as it was shown to do in the 1D ZEUS simulations of effervescent heating by Ruszkowski \& Begelman (2002). We 
also expect energetically "equivalent" bubbles to grow to more rapidly in $2 \mathrm{D}$ than in $3 \mathrm{D}$. Thus it is not surprising that the bubbles in our 2D simulations are larger than the X-ray holes at the center of the Perseus Cluster, despite our attempt to roughly match conditions. We decided to carry out two-dimensional simulations first because they are computationally much less demanding and allow us to explore a wider range of parameters. However, we are planning to report a limited set of 3D simulations separately. Preliminary results suggest that main conclusions drawn from the three-dimensional simulations are consistent with those obtained from 2D simulations.

In summary, we have demonstrated that viscous heating by an intermittent AGN located at the center of a cooling flow cluster can balance radiative cooling and, thus, quench the cooling flow. Energy is transferred to the gas by viscous dissipation of waves produced by intermittent AGN activity with a duty cycle much shorter than the cooling time. In the proposed heating mechanism, heating is gentle, spatially-distributed in a symmetric fashion and delivered to the gas located within the cooling radius faster than the cooling timescale. In this first attempt to simulate the effects of dissipation of waves in the ICM, we have assumed Spitzer viscosity, but we have to concede that the value of viscosity in the ICM is poorly constrained. Nevertheless, our results show that this heating mechanism is broadly consistent with the assumptions of the effervescent heating model (Begelman 2001; Ruszkowski \& Begelman 2002), in which dissipation of waves plays an important role (Begelman 2003), and can be applied to recently reported observations of ripples in the Perseus (Fabian et al. 2003a,b) and Virgo (Forman et al. 2003) clusters.

We thank the anonymous referee for many useful comments and Phil Armitage for his words of wisdom. In particular, the anonymous referee is acknowledged for his/her contribution to the discussion of the conversion of the input energy to the energy in the ICM (paragraph 6 in section 3). We are grateful to Andy Fabian and Chris Reynolds for their comments, which improved the paper. We also thank Peter Ruprecht and Mark Tamisiea for technical support. The software used in this work was in part developed by the DOE-supported ASCI/Alliance Center for Astrophysical Thermonuclear Flashes at the University of Chicago. We acknowledge support from the W. M. Keck Foundation, which purchased the JILA 74-processor Keck Cluster. Some of the calculations presented in this work were performed at National Center for Supercomputing Applications at the University of Illinois at UrbanaChampaign, which is funded through the PACI Program at the National Science Foundation. Support for this work was provided by National Science Foundation grant AST0307502 and the National Aeronautics and Space Administration through Chandra Fellowship Award Number PF340029 issued by the Chandra X-ray Observatory Center, which is operated by the Smithsonian Astrophysical Observatory for and on behalf of the National Aeronautics and Space Administration under contract NAS8-39073.

\section{REFERENCES}

Bowers, R.L., \& Wilson, J.R. 1991, in Numerical Modeling in Applied Physics and Astrophysics, Jones and Barlett Publishers, Inc., Boston

Balbus, S.A., Hawley, J.F., \& Stone, J.M. 1996, ApJ, 467, 76

Batchelor, G.K. 1967, An Introduction to Fluid Dynamics, Cambridge University Press, Cambridge

Begelman, M.C. 2001, in ASP Conf. Proc., 240, "Gas and Galaxy Evolution", ed. J.E. Hibbard, M.P. Rupen, \& J.H. van Gorkom (San Francisco: ASP), 363

Begelman, M.C. 2003, in Carnegie Observatories Astrophysics Series, Vol. 1: "Coevolution of Black Holes and Galaxies" ed. L. C. Ho (Cambridge: Cambridge Univ. Press), in press, astro$\mathrm{ph} / 0303040$

Blanton, E.L., Sarazin, C.L., McNamara, B.R., \& Wise, M.W. 2001, ApJ, 558, 15

Braginskii, S.L. 1958, Sov. Phys. JETP, 6, 358

Brüggen, M. 2003, ApJ, 592, 839

Brüggen, M., \& Kaiser, C.R. 2002, Nature, 418, 301

Brüggen, M., Kaiser, C.R., Churazov, E., \& Enßlin, T.A. 2002, MNRAS, 331, 545

Burns, J.O. 1990, AJ, 99, 14

Churazov, E., Brüggen, M., Kaiser, C.R., Böhringer, H., \& Forman, W. 2001, ApJ, 554, 261

Churazov, E., Sunyaev, R., Forman, W., \& Böhringer H. 2002, MNRAS, 332, 729

Croom, S. at al. 2003, in ASP Conf. Proc., "AGN Physics with the Sloan Digital Sky Survey", ed. G. T. Richards and P. B. Hall (San Francisco: ASP), in press, astro-ph/0310533

Ensslin, T.A. \& Heinz, S. 2002, A\&A, 384, 27

Fabian, A.C., et al. 2000, MNRAS, 318, 65

Fabian, A.C., Celotti, A., Blundell, K.M., Kassim, N.E., \& Perley, R.A. 2002, MNRAS, 331, 369

Fabian, A.C., Sanders, J.S., Allen, S.W., Crawford, C.S., Iwasawa, K., Johnstode, R.M., Schmidt, R.W., \& Taylor, G.B. 2003a, MNRAS, 344, 43
Fabian, A.C., Sanders, J.S., Crawford, C.S., Conselice, C.J., Gallagher, J.S., \& Wyse, R.F.G. 2003b, MNRAS, 344, 48

Forman, W. et al. 2003, submitted to ApJ, astro-ph/0312576

Hansen, H.H. \& Stadel, J. 2003, ApJ, 595, 37

Kaiser, C.R., Dennet-Thorpe, J., \& Alexander, P. 1997, MNRAS, 292,723

Landau, L.D \& Lifshitz, E.M. 1975, Fluid Mechanics, Pergamon Press

Mazzotta, P., Kaastra, J. S., Paerels, F. B., Ferrigno, C., Colafrancesco, S., Mewe, R., \& Forman, W. R. 2002, ApJ, 567, 37

McNamara, B.R., et al. 2000, ApJ, 534, 135

Navarro, J., Frenk, C.S., \& White, S.D.M. 1995, MNRAS, 275, 720

Navarro, J., Frenk, C.S., \& White, S.D.M. 1997, ApJ, 490, 493

Peterson, J.R., Paerels, F.B.S., Kaastra, J.S., et al. 2001, A\&A, 365, 104

Peterson, J.R., Kahn, S.M., Paerels, F.B.S., Kaastra, J.S., et al. 2003, ApJ, 590, 207

Porter, D.H., \& Woodward, P.R. 1994, ApJS, 93, 309

Pringle, J.E. 1989, MNRAS, 239, 479

Quilis, V., Bower, R., \& Balogh, M.L. 2001, MNRAS, 328, 1091

Reynolds, C.S., Heinz, S., \& Begelman, M.C. 2001, ApJ, 594, 197

Ruszkowski, M., \& Begelman, M.C. 2002, ApJ, 581, 223

Shu, F.H., 1992, The Physics of Astrophysics, Vol.2, Gas Dynamics, University Science Books, Sausalito, CA

Sutherland, R.S., \& Dopita, M.A. 1993, ApJS, 88, 253

Sytine, I.V., Porter, D.H., Woodward, P.R., Hodson, S.W., \& Winkler, K.-H. 2000, J. Comput. Phys., 158, 225

Tabor, G., \& Binney, J. 1993, MNRAS, 263, 323

Tamura, T., Kaastra, J.S., Peterson, J.R., Paerels, F., et al. 2001, A\&A, 365, 87

Tozzi, P., \& Norman, C. 2001, ApJ, 546, 63 


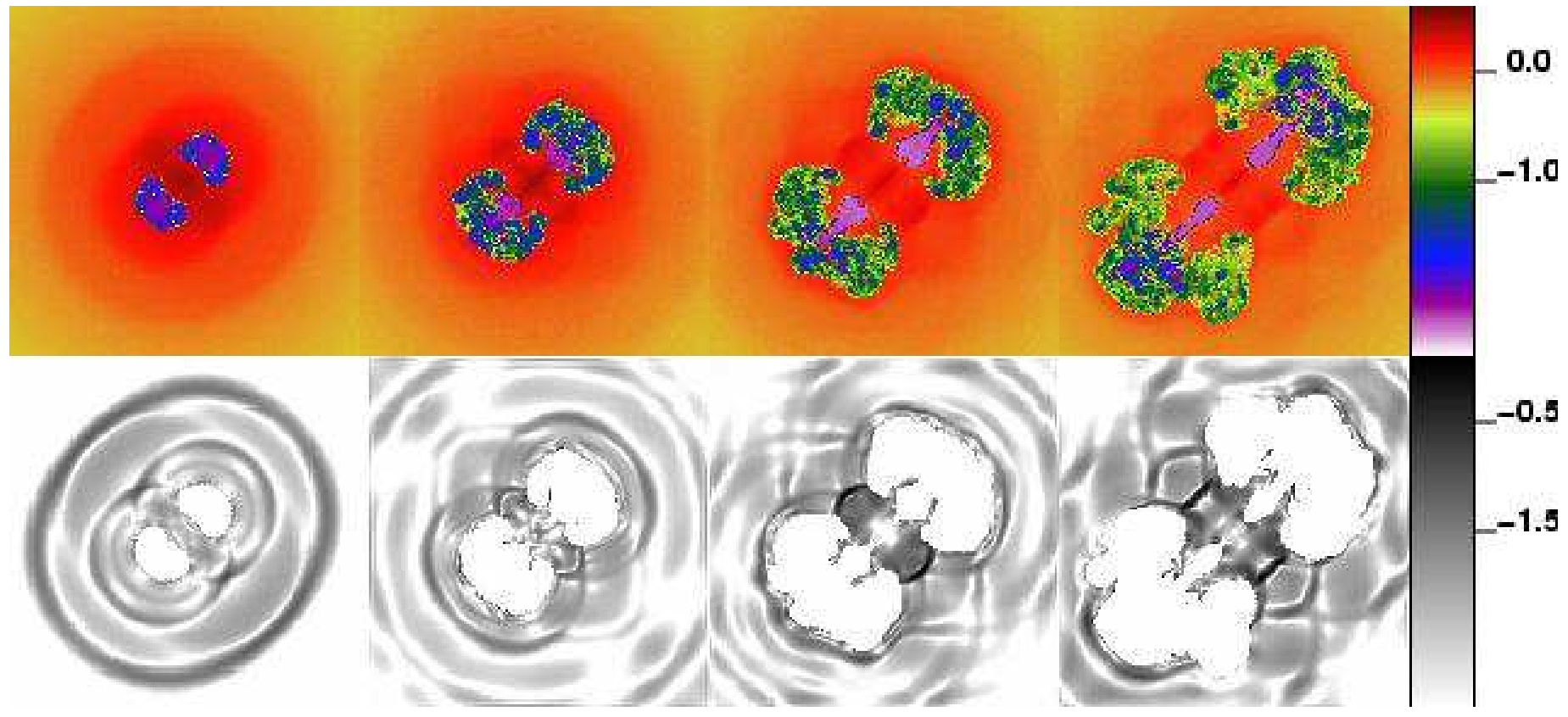

FIG. 1. - Top panels show the evolution of density due to intermittent energy injection near the cluster center. Unit mass density in code units corresponds to a real electron number density of $8.75 \times 10^{-3} \mathrm{~cm}^{-3}$. Bottom panels show viscous energy dissipation rate. All plots are in logarithmic scale. Unit energy dissipation rate per unit mass in code units corresponds to $3.24 \mathrm{~cm}^{2} \mathrm{~s}^{-} 3$. Snapshots correspond to $5.8 \times 10^{7}$, $1.2 \times 10^{8}, 1.8 \times 10^{8}$ and $2.3 \times 10^{8}$ years, respectively. The source is periodically active for $1.5 \times 10^{7}$ years and dormant for $1.5 \times 10^{7}$ years. 


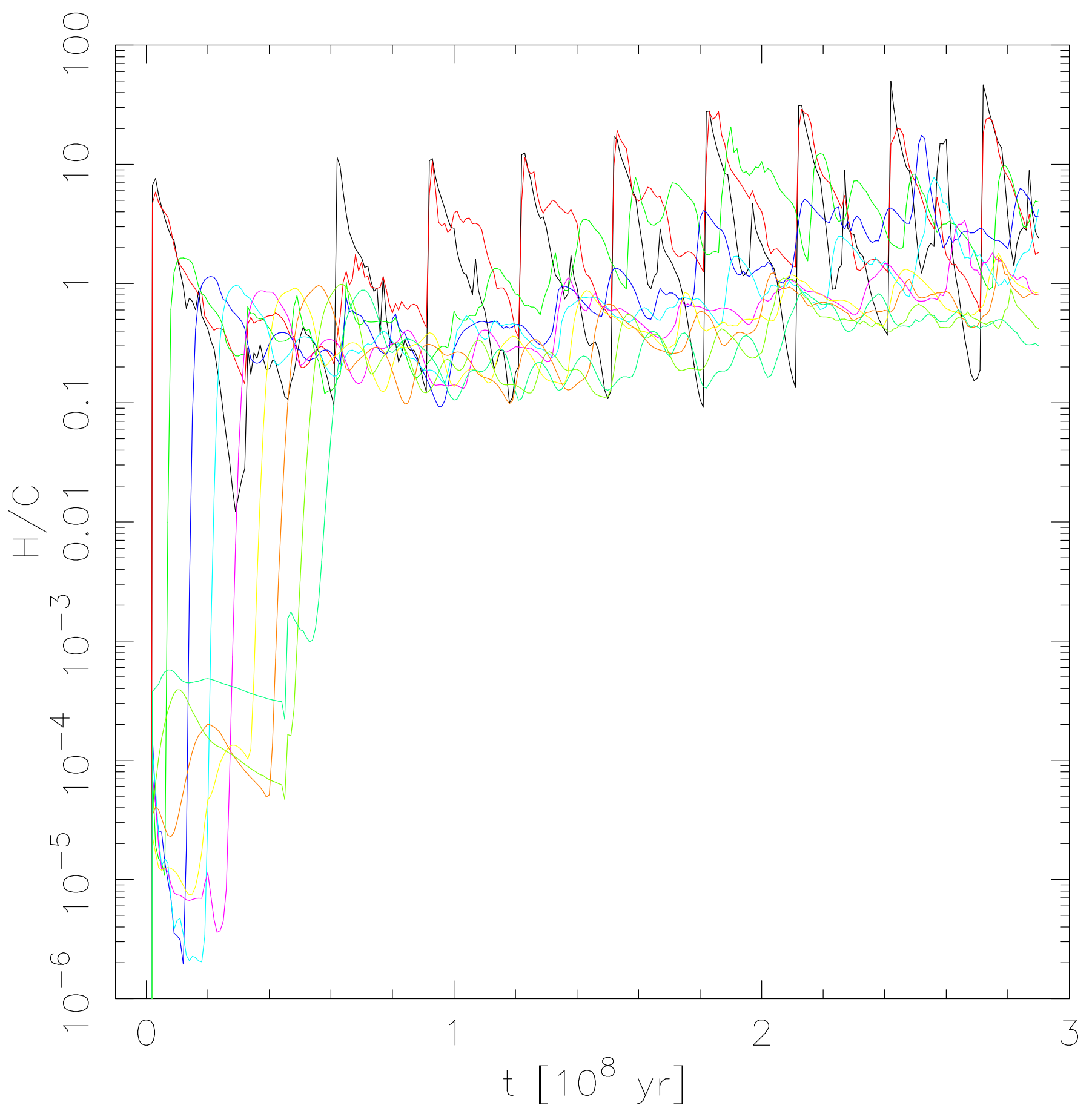

FIG. 2.- The ratio of viscous heating to radiative cooling rate as a function of time for a number of concentric annuli around the cluster center. The curves that start rising at later times correspond to annuli located further away from the center. The heating-to-cooling ratio was calculated in ten rings starting from the first ring at $5 \mathrm{kpc}$ and the remaining rings located in increments of $10 \mathrm{kpc}$ away from the cluster center. Note that the heating rate is comparable to the cooling rate. Note also that the curves display a pronounced periodic behavior that reflects the intermittency of the central source. 